\title{
RELACIONES ENTRE NIVEL DE DESARROLLO MORAL E INTELECTUAL, EDAD Y GENERO EN ALUMNOS DE SECUNDARIA DE UN COLEGIO EXPERIMENTAL/ALTERNATIVO DE LIMA
}

\author{
Manuel Bello Domínguez ${ }^{1}$ \\ Eduardo Mejía Carbonell ${ }^{2}$
}

Se aplicaron las pruebas SROM (nivel de juicio moral) y de LONGEOT (nivel de desarrollo intelectual) a 34 alumnos/as de educación secundaria del colegio experimental/altemativo "José Antonio Encinas" de Lima con edad entre 12 y 17 años.

Los resultados muestran que existen marcadas diferencias entre los resultados encontrados y los de estudios anteriores realizados tanto en el país como en el extranjero. Los sujetos estudiados presentan niveles intelectuales más altos que los del promedio e incluso mayores que los hallados en muestras de personas de mayor edad, alcanzando la gran mayoría de ellos los niveles de pensamiento formal. En relación al juicio moral ocurre algo similar, pues la mayoría de ellos habían logrado un nivel maduro, pese a su corta edad. Se encuentran correlaciones altas y positivas entre los niveles intelectuales y de juicio moral. También se encuentran correlaciones altas y positivas entre nivel de pensamiento y edad, $y$ entre nivel de juicio moral y edad. No se encontraron diferencias de género en los niveles de pensamiento y de juicio moral.

Se discute la probable relación entre los resultados encontrados y las características del modelo de educación alternativa aplicado en el colegio "Encinas", que prioriza el desarrollo de habilidades y actitudes y asigna a los propios alumnos un papel activo y protagónico en el proceso educativo.
The Sociomoral Reflection Objective Measure (SROM) and the LONGEOT Intelligence Test were administered to 34 high school students aged between 12 and 17 years from an experimental school of Lima.

Significant differences were found between results of this study and those of previous studies carried out in Peru and in USA. The students showed intellectual developments above the mean, most of them having achieved Piaget's Formal Thought. With regard to moral judgement, similar results were observed. The majority of sudents had achieved Kolhberg's Conventional Level. Positive correlations were found between age and thought development and between moral judgement and age. No significant differences between genders were observed.

Results are discussed taking into account the characteristics of the school curriculum which emphasizes the development of abilities, attitudes and active participation of their students.

1. Maestro en Ciencias con mención en Psicología. Profesor Asociado del Departamento de Psicología, Universidad Peruana Cayetano Heredia. Director del Centro de Investigación y Desarrollo Integrante de "Foro Educativo". Dirección postal: Parque Hernán Velarde N 72, Santa Beatriz, Lima 1. Telf. 333762.

2. Bachiller en Psicología, Universidad Peruana Cayetano Heredia. 



\section{Introducción}

Investigar en la actualidad sobre el desarrollo moral e intelectual de los niños y jóvenes peruanos es de gran importancia. La crisis que vive nuestro país tiene una fuerte relación con el poco interés del sistema educativo nacional por desarrollar los aspectos valorativo-actitudinales y cognitivos de los alumnos y alumnas.

Las deficiencias de la concepción de educación predominante en el Perú y muchos otros países han sido señaladas por educadores y psicólogos de todas las tendencias (Encinas, 1932; Neill, 1967; Holt, 1982; Skinner, 1978; Piaget, 1973; Ferreiro, 1990; etc.).

En el Perú el propio Ministerio de Educación ha recogido las críticas que relacionan al modelo pedagógico vigente con los problemas de cobertura y de calidad de la educación nacional: la deformación de la enseñanza-aprendizaje expresada en el exceso de la exigencia memorística; la conversión de las clases en sesiones de copiado de materiales pre-hechos o del libro; la escasa confiabilidad de la evaluación y la excesiva importancia de la nota como factor de motivación a los alumnos, etc. (Ministerio de Educación, 1986, p. 22). También existen referencias a las consecuencias negativas de la educación escolar en el desarrollo afectivo y moral de nifnos/as y jóvenes (Gonzales M., 1990).

La llamada "educación alternativa" busca ser una respuesta teórica y metodológica a las severas deficiencias antes mencionadas y ante la urgencia de contar con una educación que contribuya a la democratización y al desarrollo económico y social del país. Es una propuesta que prioriza el desarrollo de habilidades (de investigación, de comunicación, creativas, motoras, manuales, sociales, etc.) y actitudes (solidaridad, justicia, criticidad, creatividad, etc.), y en la cual los alumnos/as son protagonistas de un proceso que enfatiza el aprender desde y para la realidad actual. (Bello, 1990).

El Programa Educativo "José Antonio Encinas" es uno de los colegios de Lima que realiza, desde 1982, una experiencia institucional de educación al- 
ternativa. En este Programa las asignaturas han sido cambiadas por un régimer de "actividades", en las cuales los alumnos realizan tareas de investigaciór. (revisión de libros, revistas, periódicos y otras fuentes; observación, descripción, encuestas, experimentos, etc.), y la producción de materiales, textos. objetos artísticos y artesanales, entre otros. Los mecanismos represivos de control y las relaciones autoritarias han sido reemplazados por una organización participativa y personalizada de la convivencia basada en la autorregulación del comportamiento individual y colectivo (Arellano, 1985; Dammert, 1985; Bello. 1990).

El propósito del presente estudio es doble. En primer lugar observar de qué modo la experiencia de educación alternativa influye sobre el desarrollo actitudinal-valorativo e intelectual de sus alumnos y comparar los resultados con los de otros estudios similares realizados en el Perú y en el extranjero. En segundo término, a un nivel teórico, confirmar la hipotética relación entre el nivel de desarrollo moral e intelectual y la asociación entre el desarrollo en ambos aspectos y la edad; también verificar la ausencia de diferencias significativas entre los géneros.

\section{Antecedentes}

El estudio del desarrollo moral e intelectual en el presente trabajo se ha realizado en el marco del enfoque cognitivo-evolutivo: la teoría de Kohlberg sobre el desarrollo del juicio moral (Hersch et al., 1984) y la teoría de Piagel sobre el desarrollo del pensamiento (Delval, 1979).

Para Piaget la inteligencia opera a nivel afectivo y viceversa: el afecto motiva las operaciones del conocimiento y el conocimiento estructura las operaciones del afecto. Piaget empezó a investigar el juicio moral a partir de su estudio del desarrollo en los niños del respeto de las reglas de juego y del sentido de solidaridad con su sociedad. A partir de esos estudios y otros posteriores se elaboro una teorfa del desarrollo moral, que identifica tres períodos: moral heterónoma, sociónoma y autónoma (Misfud, 1985).

Kohlberg, basándose en Piaget, construye una teoría del desarrollo socioafectivo utilizando el concepto de "juicio moral", entendiéndolo como un modo de evaluación prescriptivo de lo bueno y de lo recto" (Hersh et al., 1984). Para este autor el "principio de justicia" es el criterio universal y básico de la moralidad y consiguientemente el factor básico en el desarrollo del juicio moral; el desarrollo moral se realiza en una secuencia de seis estadíos, divididos en tres niveles; en este proceso el individuo consigue cada vez mayor diferenciación e integración en su juicio moral y mayor equilibrio psicosocial. 
Lo niveles y estadíos planteados por Kolhberg son:

1. Nivel preconvencional: El niño responde a las reglas culturales y a lo que se denomina bueno y malo, en función de las consecuencias físicas o hedonísticas de su acción y/o del poder físico de aquellos que establecen las reglas. Comprende dos estadíos:

- Estadío 1: La orientación del castigo y obediencia.

- Estadío 2: La orientación instrumental y relativista.

II. Nivel convencional: El respeto de las expectativas de la familia, el grupo o la nación es un valor en sí mismo que se asume sin tomar en cuenta las consecuencias inmediatas y obvias. Se dan los siguientes estadíos:

- Estadío 3: La concordancia interpersonal o la orientación del "buen chico".

- Estadío 4: La orientación de la ley y del orden.

III. Nivel post-convencional: Hay un esfuerzo para definir valores y principios morales que tengan validez y aplicación universal, incluso por encima de la autoridad de los grupos o las personas con los que cada uno se identifica:

- Estadío 5: La orientación legalista del contrato social.

- Estadío 6: La orientación por principios universales.

Estudios realizados en muchos lugares del mundo han demostrado la validez universal y transcultural de esta secuencia de desarrollo del juicio moral formulada por Kohlberg (Snarey, 1985; Nisan y Kohlberg, 1982).

De otro lado, la teoría de Piaget acerca del desarrollo de la inteligencia en el ser humano establece los estadíos del desarrollo cognitivo desde la infancia a la adultez: describe y explica cómo las estructuras psicologicas se desarrollan a partir de los reflejos innatos, se organizan durante la infancia en esquemas de conducta, se internalizan durante el segundo año de vida como modelos de pensamiento, y se desarrollan durante la infancia y la adolescencia en las complejas estructuras intelectuales que caracterizan la vida adulta. Piaget divide este desarrollo en cuatro períodos: sensoriomotor (del nacimiento a los 2 años); preoperatorio (de 2 a 7 años); operaciones concretas (de 7 a 11 años); y operaciones formales (de los 12 en adelante) (Delval, 1979).

El período de las operaciones concretas se refiere a las operaciones lógicas que realizan los niños sobre objetos concretos. Se llaman "operaciones" a las acciones mentales reversibles, tales como las sumas y las restas. Se les llama "concretas" porque los niños de esta edad generalmente piensan las cosas en términos de objetos concretos y operan con ellos. 
El periodo de las operaciones formales se caracteriza por la capacidad para razonar en términos abstractos, "hacer operaciones sobre operaciones". Una vez que se entiende que los objetos se pueden clasificar por criterios formales, lógicamente, se puede empezar sistemáticamente a comparar y contrastar varios objetos con esos criterios. Además, lo dado se puede ver en relación a otras posibilidades hipotéticas. Este período cubre diversos grados de capacidad para pensar en abstracto.

La educación secundaria peruana está planteada normativamente para la etapa de los 12 a 16 años, en la cual se debe alcanzar el nivel convencional del juicio moral (estadíos 3 y 4 de Kohlberg), que es el nivel "maduro" exhibido por la generalidad de los adultos (Gibbs et al., 1984; Majluf, 1986), y se debe producir el tránsito al pensamiento hipotético-deductivo y las operaciones formales.

Gibbs et al. en 1984 aplicaron en Estados Unidos el SROM (Sociomoral Reflection Objective Measure) para establecer el nivel de desarrollo del juicio moral, encontrando para los sujetos de 14 años un puntaje promedio de 327.98 -que corresponde al estadío 3(4)-, y para los de 16 años un puntaje promedio de 355.13 que equivale al estadío 4(3). Majluf (1986) replicó dicho estudio en el Perú encontrando resultados similares en la población de nivel socio-económico medio.

Valdez (1985) realizó un estudio similar en sujetos de tres edades diferentes $(8,11$ y 16 años) en ambos sexos y en dos estratos socio-económicos (bajo y alto) no hallando diferencias significativas de género y clase social. Encontró que los sujetos de 11 años se ubicaban en promedio en el nivel 2 y los de 16 años en el nivel 3(2); los del estrato socio-económico alto alcanzaban en promedio el nivel 3.

En un estudio realizado en maestras de Educación Inicial de Lima (Montenegro, 1987) se halló que la mayoría de sujetos de la muestra $(88,33 \%)$ se ubicaban en el nivel 4. Se encontró diferencias entre los sujetos menores de 25 años, los de 25 a 35, y los de más de 35 años: a menor edad era mayor el nivel intelectual y del juicio moral.

Basinger, Biggs y Fuller (1992) al validar un nuevo instrumento de medición del juicio moral basado en la teoría de Kohlberg, encontraron niveles promedio de 3(2) para los sujetos de 14 años y de 3 para los de 17 años. Sólo los sujetos considerados adultos alcanzaban el nivel 4(3).

En lo que se refiere al desarrollo del pensamiento, Majluf (1974) realizo un estudio en alumnos del quinto de secundaria de ambos sexos (con una edad media de 18 años) utilizando la prueba de Longeot. Los resultados obtenidos 
con el Test de Operaciones Lógicas Proposicionales fueron: en colegios de los pueblos jóvenes (nivel socio-económico bajo) sólo 30\% de los alumnos alcanzaron el nivel de pensamiento lógico formal, mientras que en colegios particulares (sector medio) esto fue logrado por el $72 \%$ de los alumnos,

De otro lado, Aliaga en 1978 evaluó con instrumentos piagetanos a sujetos de 15 a 46 años, de los cuales sólo un $23 \%$ alcanzaron el nivel de pensamiento lógico-formal.

Montenegro (1987) encontró que el $46,7 \%$ de las maestras de su estudio se ubican en el nivel concreto de pensamiento y el 53,3\% restante lograban un pensamiento abstracto, de acuerdo a los resultados del test de operaciones combinatorias de Longeot.

\section{El estudio realizado}

Los objetivos del estudio fueron:

1. Determinar el nivel de juicio moral (Kohlberg) y de desarrollo del pensamiento (Piaget) de los alumnos/as de secundaria del colegio "Encinas".

2. Determinar el grado de correlación entre el nivel intelectual, el juicio moral, la edad y el sexo.

3. Comparar los resultados con los obtenidos en otros estudios similares.

El estudio se hizo con el propósito de responder y contrastar las siguientes preguntas e hipótesis especificas:

1. ¿Cuál es y cómo se distribuye el nivel de juicio moral (Kohlberg) de los alumnos/as de secundaria del colegio "José A. Encinas"?

2. ¿Cuál es y cómo se distribuye el nivel de pensamiento (Piaget) de los alumnos/as de secundaria del colegio "José A. Encinas"?

3. ¿Existe correlación entre el nivel de pensamiento y el del juicio moral en el grupo de alumnos/as a estudiar?

Ha: "Existe correlación positiva entre el nivel de pensamiento y el nivel de juicio moral".

4. ¿Existe correlación entre el nivel del pensamiento y la edad en el grupo de alumnos/as a estudiar?

Ha: "Existe correlación positiva entre el nivel de pensamiento y la edad". 
5. ¿Existe correlación entre el nivel de juicio moral y la edad en el grupo de alumnos/as a estudiar?

Ha: "Existe correlación positiva entre el nivel de juicio moral y la edad".

6. ¿Existen diferencias significativas en el nivel de pensamiento de varones y mujeres en el grupo de alumnos/as a estudiar?

Ha: "No se encontrarán diferencias significativas entre varones y mujeres en el nivel de pensamiento?

7. ¿Existen diferencias significativas en el nivel de juicio moral de varones y mujeres en el grupos de alumnos/as a estudiar?

Ha: "No se encontrarán diferencias significativas entre varones y mujeres en el nivel de juicio moral".

\section{Método}

Sujetos: El nivel de educación secundaria de este Colegio contaba (en 1991) con 53 alumnos de ambos sexos (24 mujeres y 29 varones) que estaban repartidos como sigue: 18 alumnos en el primer año de secundaria, 18 alumnos en el segundo año y 17 alumnos entre el cuarto y quinto de secundaria. Debido a las particularidades de la enseñanza los estudiantes del cuarto y quinto de secundaria estudiaban en un mismo salón y desarrollaban el mismo programa.

Los estratos socioeconomicos predominantes son medio y medio-bajo, siendo la mayoría de los padres profesionales y/o trabajadores independientes.

De los 53 alumnos, siete fueron descartados por su inasistencia al colegio los días de la evaluación y $12(26,1 \%$ de 46$)$ invalidaron la prueba SROM al superar la cantidad máxima de respuestas "pseudo" permitidas según las normas de calificación de la prueba ${ }^{3}$. En consecuencia, el número total de sujetos considerados en el estudio se redujo a 34 . La distribución de estos según edad y género se presenta en el Cuadro 1:

Instrumentos: Para este estudio se utilizaron las siguientes pruebas:

- La Escala de Reflexión Moral (en inglés original "Sociomoral Reflection Objetive Measure" o "SROM") creada por Gibbs y Widaman, basada en la teoría de Kohlberg. Esta prueba permite hacer mediciones colectivas del

3. El porcentaje es bajo si se compara con el registrado en otros estudios. Por ejemplo Montenegro (1987) reporta $40 \%$ de pruebas invalidadas con sujetos de 25 a 40 años de edad. 
desarrollo moral ${ }^{4}$. La confiabilidad y validez del SROM están verificadas para la mayoría de la población adolescente y adulta (estudio realizado en 1984 en Estados Unidos).

\section{Cuadro 1}

Muestra de Estudiantes

\begin{tabular}{lccc}
\hline Edades & Hombres & Mujeres & Total \\
\hline 12 & 2 & 6 & 8 \\
13 & 6 & 3 & 9 \\
14 & 1 & 3 & 4 \\
15 & 3 & 2 & 5 \\
16 & 3 & 2 & 5 \\
17 & 2 & 1 & 3 \\
\hline Total & 17 & 17 & 34 \\
\hline
\end{tabular}

La prueba SROM propone, al igual que sus antecedentes SRM y el MJI, el análisis de dilemas morales cuyas respuestas se califican según la Escala de Juicio Moral de Kohlberg. A diferencia de las pruebas mencionadas, en el SROM las respuestas deben escogerse entre 5 alternativas válidas más una pseudoaltemativa supernumeraria, las que se traducen en puntajes. El cómputo en el nivel de "madurez" que le corresponde.

- La Escala de Nivel Intelectual del Longeot, creada entre 1960-61 y validada entre los años 1962-65. Para este trabajo se ha utilizado una de las tres pruebas originales de la Escala: el Test de las Operaciones Formales de Lógica de las Proposiciones (TOFLP). La prueba se creo basada en la teoria de Piaget y con el proposito de medir el nivel de desarrollo cognoscitivo en forma colectiva. Presenta niveles de validez y confiabilidad para población adolescente y adulta (validado entre 1962-1965).

Procedimiento. Se administraron las pruebas colectivamente en las tres aulas. Se aplicó en primer lugar la prueba de LONGEOT y dos días después la prueba SROM.

\section{Resultados}

En el siguiente cuadro se presentan los resultados generales del estudio:

4. Oras formas de medir son el MJI ("Moral Judgement Interview", creado por el mismo Kohlberg) por medio de entrevista personal o el SRM ("Sociomoral Reflection Measures", de Gibbs) que es de aplicación individual. 
Cuadro 2

Resultados generales

\begin{tabular}{|c|c|c|c|c|c|c|}
\hline \multirow{2}{*}{\multicolumn{2}{|c|}{$\begin{array}{c}\text { Sujetos } \\
\text { (Edad-Sexo) }\end{array}$}} & \multicolumn{2}{|c|}{ SROM } & \multirow{2}{*}{$\begin{array}{l}\text { Longeot } \\
\text { Puntaje }\end{array}$} & \multirow{2}{*}{\multicolumn{2}{|c|}{$\begin{array}{c}\text { (TOFLP) } \\
\text { Nivel }\end{array}$}} \\
\hline & & \multirow{2}{*}{$\begin{array}{c}\text { Puntaje } \\
365\end{array}$} & \multirow{2}{*}{$\frac{\text { Nivel }}{4(3)}$} & & & \\
\hline 1. & $(12, M)$ & & & (7) & IIIA & $\mathbf{F}$ \\
\hline 2. & $(13, F)$ & 316 & 3 & (7) & IIIA & $\mathbf{F}$ \\
\hline 3. & $(12, F)$ & 357 & $4(3)$ & (4) & II & C \\
\hline 4. & $(13, F)$ & 400 & 4 & (6) & IIIA & $\mathbf{F}$ \\
\hline 5. & $(13, M)$ & 262 & $3(2)$ & (6) & IIIA & $\mathbf{F}$ \\
\hline 6. & $(12, F)$ & 279 & 3 & (2) & II & $\mathrm{C}$ \\
\hline & $(12, M)$ & 315 & 3 & (3) & II & $\mathrm{C}$ \\
\hline 8. & $(14, F)$ & 397 & 4 & (7) & IIIA & $\mathbf{F}$ \\
\hline & $(12, F)$ & 248 & 3 & (7) & IIIA & $\mathbf{F}$ \\
\hline 10. & $(14, F)$ & 335 & $3(4)$ & (7) & IIIA & $F$ \\
\hline & $(13, M)$ & 366 & $4(3)$ & (7) & IIIA & $F$ \\
\hline & $(14, M)$ & 303 & 3 & (6) & IIIA & $\mathrm{F}$ \\
\hline 13. & $(14, F)$ & 413 & 4 & (4) & II & $\mathrm{C}$ \\
\hline & $(13, M)$ & 383 & 4 & (6) & IIIA & $\mathrm{F}$ \\
\hline 15. & $(13, M)$ & 350 & $4(3)$ & (9) & IIIB & $\mathbf{F}$ \\
\hline 16. & $(12, F)$ & 407 & 4 & (7) & IIIA & $\mathbf{F}$ \\
\hline 17. & $(13, M)$ & 373 & $4(3)$ & (7) & IIIA & $F$ \\
\hline 18. & $(12, F)$ & 333 & $3(4)$ & (9) & IIIB & $\mathbf{F}$ \\
\hline & $(13, M)$ & 386 & 4 & (8) & IIIA & $\mathrm{F}$ \\
\hline 20. & $(13, F)$ & 325 & 3 & (5) & IIIA & $\mathrm{F}$ \\
\hline 21. & $(12, F)$ & 334 & $3(4)$ & (6) & IIIA & $\mathrm{F}$ \\
\hline & $(15, M)$ & 436 & 4 & (10) & IIIB & $\mathbf{F}$ \\
\hline 23. & $(15, M)$ & 396 & 4 & (10) & IIIB & $\mathbf{F}$ \\
\hline 24. & $(16, F)$ & 367 & $4(3)$ & (4) & II & $\mathrm{C}$ \\
\hline & $(16, M)$ & 431 & $4(5)$ & (8) & IIIB & $\mathbf{F}$ \\
\hline 26. & $(15, F)$ & 471 & $5(4)$ & (8) & IIIB & $\mathbf{F}$ \\
\hline & $(15, M)$ & 440 & $4(5)$ & (8) & IIIB & $\mathrm{F}$ \\
\hline & $(16, F)$ & 449 & $4(5)$ & (8) & IIIB & $\mathbf{F}$ \\
\hline & $(17, M)$ & 378 & 4 & (7) & IIIA & $\mathbf{F}$ \\
\hline & $(17, F)$ & 395 & 4 & (9) & IIIB & F \\
\hline & $(17, \mathbf{M})$ & 426 & $4(5)$ & (6) & IIIA & F \\
\hline & $(16, M)$ & 453 & $5(4)$ & (7) & IIIA & $\mathbf{F}$ \\
\hline & $(16, M)$ & 369 & $4(3)$ & (8) & IIIB & $\mathbf{F}$ \\
\hline 34. & $(15, F)$ & 381 & 4 & (9) & IIIB & $\mathbf{F}$ \\
\hline
\end{tabular}




\section{Análisis de los resultados y verificación de hipótesis}

1. Sobre el nivel de juicio moral

En el Cuadro 3 se muestran los resultados del SROM teniendo el promedio de sus puntajes en relación al género en cada grupo etario.

\section{Cuadro 3}

Resultados del SROM

\begin{tabular}{|c|c|c|c|c|c|c|}
\hline \multirow[b]{2}{*}{ Edades } & \multicolumn{3}{|c|}{ Hombres } & \multicolumn{3}{|c|}{ Mujeres } \\
\hline & $\mathrm{N}$ & Ptje. $\bar{X}$ & Nivel & $\mathbf{N}$ & Ptje. $\bar{X}$ & Nivel \\
\hline 12 & 2 & 340 & $3(4)$ & 6 & 326 & $3(4)$ \\
\hline 13 & 6 & 351.2 & $4(3)$ & 3 & 320.5 & 3 \\
\hline 14 & 1 & 303 & 3 & 3 & 381.6 & 4 \\
\hline 15 & 3 & 424 & 4 & 2 & 426 & $4(5)$ \\
\hline 16 & 3 & 411 & 4 & 2 & 408 & 4 \\
\hline \multirow[t]{2}{*}{17} & 2 & 402 & 4 & 1 & 395 & 4 \\
\hline & 17 & 374.07 & $4(3)$ & 17 & 360.3 & $4(3)$ \\
\hline
\end{tabular}

Los promedios generales del cuadro muestran que los sujetos han alcanzado el nivel maduro de juicio oral .Además, vemos que en el Cuadro 2 el 70.58\% (24 alumnos) alcanzaron el nivel maduro (de nivel 4 para adelante).

2. Sobre el nivel de desarrollo del pensamiento

Se observa en el Cuadro 4 el número de sujetos en cada nivel de pensamiento según la prueba TOFLP de Longeot.

\section{Cuadro 4}

Resultados de la Prueba de LONGEOT

\begin{tabular}{l|cc|cc}
\hline Nivel de & \multicolumn{2}{|c|}{ Concreto } & \multicolumn{2}{c}{ Formal } \\
Pensamiento & IC & IIC & IIIA & IIIB \\
(TOFLP) & & & & \\
\hline No de Sujetos & - & 5 & 18 & 11 \\
\hline Porcentaje & $0 \%$ & $14.41 \%$ & $52.94 \%$ & $32.36 \%$ \\
\hline
\end{tabular}

Se observa que más del $85 \%$ de los sujetos alcanzaron el nivel de pensamiento formal en la prueba de lógica de las proposiciones. Por lo tanto se puede afirmar que la gran mayoría de los alumnos/as del grupo estudiado operan con un pensamiento abstracto. 
3. Correlación entre nivel de pensamiento y juicio moral

En el Cuadro 5 se relaciona el nivel de pensamiento (TOFLP) con el nivel del juicio moral.

\section{Cuadro 5}

Relación entre el Nivel de Pensamiento y Juicio Moral

\begin{tabular}{l|cc|rr|r}
\hline \multicolumn{5}{|c|}{ Nivel de pensamiento (TOFLP) } \\
\hline Nivel & \multicolumn{2}{|c|}{ Concreto } & \multicolumn{2}{|c}{ Formal } & \multirow{2}{*}{ Moral } \\
\hline \multirow{2}{*}{ IC } & IIC & IIIA & IIIB & Total \\
\hline$(3)$ & & 2 & 7 & 1 & 11 \\
$(4)$ & & 3 & 10 & 9 & 22 \\
$(5)$ & & & 1 & 1 & 2 \\
\hline Total & - & 5 & 18 & 11 & 34 \\
\hline
\end{tabular}

En los puntajes de todos los sujetos de la muestra válida se encontró una correlación de $\mathrm{r}=\mathbf{0 . 8 0}$ entre el nivel moral y nivel de pensamiento. Para encontrar la correlación se utilizó el estadístico " $r$ " de Spearman. Por lo tanto, se confirma que sí existe correlación positiva entre el nivel de pensamiento y el nivel de juicio moral.

4. Correlación entre nivel de pensamiento y edad

En el Cuadro 6 se relaciona el nivel de pensamiento con la edad de los sujetos.

\section{Cuadro 6}

Nivel de Pensamiento y Edad

\begin{tabular}{|c|c|c|c|c|c|}
\hline \multicolumn{6}{|c|}{ Nivel de pensamiento (TOFLP) } \\
\hline \multirow[b]{2}{*}{ Edad } & \multicolumn{2}{|c|}{ Concreto } & \multicolumn{2}{|c|}{ Formal } & \multirow[b]{2}{*}{ Total } \\
\hline & IC & IIC & IIIA & IIIB & \\
\hline 12 & & 3 & 4 & 1 & 8 \\
\hline 13 & & & 8 & 1 & 9 \\
\hline $\begin{array}{l}14 \\
15\end{array}$ & & 1 & 3 & 5 & 5 \\
\hline 16 & & 1 & 1 & 3 & 5 \\
\hline 17 & & & 2 & 1 & 3 \\
\hline Total & - & 5 & 18 & 11 & 34 \\
\hline
\end{tabular}


La correlación hallada entre el TOFLP y la edad es de $r=0.9845$. Los resultados por tanto confirman que existe una correlación positiva entre la edad y el nivel de pensamiento, por lo que se acepta la hipótesis Ha.

5. Correlación entre nivel de juicio moral y edad

En el Cuadro 7 se presenta la relación entre el nivel de juicio moral y la edad

\section{Cuadro 7}

Nivel de Juicio Moral y Edad

\begin{tabular}{lllcc}
\hline \multicolumn{5}{c}{ Nivel de juicio moral (SROM) } \\
Edad & 3 & 4 & 5 & Total \\
\hline 12 & 5 & 3 & & 8 \\
13 & 3 & 6 & & 9 \\
14 & 2 & 2 & & 4 \\
15 & & 4 & 1 & 5 \\
16 & & 4 & 1 & 5 \\
17 & 3 & & 3 \\
\hline Total & 10 & 22 & 2 & 34 \\
\hline
\end{tabular}

La correlación entre juicio moral y edad es de $r=0.8418$, por lo tanto se acepta la hipotesis Ha.

6. Diferencias de género en el nivel de pensamiento

En el Cuadro 8 se relaciona el nivel de pensamiento con el género.

Cuadro 8

Nivel de Pensamiento y Género

\begin{tabular}{l|cccc|c}
\hline & \multicolumn{4}{|c|}{ Nivel de pensamiento (TOFLP) } & \\
Género & IC & IIC & IIIA & IIIB & Total \\
\hline Hombre & - & 1 & 10 & 6 & 17 \\
Mujer & - & 4 & 8 & 5 & 17 \\
\hline Total & - & 5 & 18 & 11 & 34 \\
\hline
\end{tabular}


Se puede observar que el número de varones que acceden a los niveles formales de pensamiento es ligeramente mayor que el de las mujeres. Sin embargo, mediante la prueba " $U$ " de Mann-Withney se encontró que Um $=99.5$ (valor de la " $U$ " en el género masculino) y Uf $=145$ (valor de " $U$ " en el género femenino); ambas son mayores que la Uc $=87$ (valor de la " $U$ " calculada) a un nivel de contraste bilateral de $a=0.05$, lo cual muestra que las diferencias no son estadísticamente significativas. Por lo tanto se acepta la $\mathrm{Ha}$, según la cual no se encontrarfan diferencias entre varones y mujeres en cuanto a nivel de pensamiento.

7. Diferencias de género en el nivel de juicio moral

En el Cuadro 9 se relaciona el nivel de juicio moral con el género.

\section{Cuadro 9}

Nivel de Juicio Moral y Género

\begin{tabular}{|c|c|c|c|c|}
\hline \multirow[b]{2}{*}{ Género } & \multicolumn{3}{|c|}{ Nivel de juicio moral } & \multirow[b]{2}{*}{ Total } \\
\hline & 3 & 4 & 5 & \\
\hline Hombre & 3 & 13 & 1 & 17 \\
\hline Mujer & 8 & 8 & 1 & 17 \\
\hline Total & 11 & 21 & 2 & 34 \\
\hline
\end{tabular}

Se observan, como en el caso anterior, diferencias de género. Mediante la prueba " $U$ " de Mann-Whitnney se encontro. Um $=110$ (valor de " $U$ " en el género masculino) y Uf - 145 (valor de "U" en el género femenino); ambas son mayores que la Uc $=87$ (valor de la " $U$ " calculada) a un nivel de contraste bilateral de $a=0.05$ lo cual muestra que tales diferencias no son estadísticamente significativas. En consecuencia se acepta la $\mathrm{Ha}$.

\section{Discusión}

Los resultados en general muestran que los alumnos de secundaria del colegio alternativo/experimental estudiados alcanzan niveles superiores a los esperados para su edad y a los encontrados en estudios previos, tanto en el desarrollo del juicio moral como en el desempeño intelectual.

Ha quedado establecido que los alumnos/as de la muestra han alcanzado un nivel maduro de desarrollo del juicio moral. Los puntajes promedio por edad en el SROM (Cuadro 3) de estos sujetos superan los puntajes de los estudios realizados por Gibbs, et al. en 1984 en USA, por Majluf en 1986 en el Perú 
(ambos estudios se llevaron a cabo con sujetos de 14, 16, 18 y 44 años), Valdez (1985) en Perú y Basinger en EE.UU. (1992).

En el estudio de Gibbs y Amold los sujetos de 14 años obtuvieron un puntaje promedio de 327.98 (nivel 3(4)) y los de 16 un puntaje de 355.13 (nivel de 4(3)). En el estudio de Valdez los sujetos de clase alta (que puntúan más alto que los de la baja) de 16 años alcanzaron el nivel 3. En el estudio de Basinger los de 14 años llegaron en promedio al nivel 3(2) y los de 17 al nivel 3 . Los sujetos del presente estudio tienen a los 14 años 348.50 (nivel 3(4)) y los de 16 tienen 413.80 (nivel 4); es decir, sus promedio son superiores a los de todos los estudios revisados.

En conjunto, el $70 \%$ de los alumnos evaluados presentan niveles de juicio moral maduros (estadíos 4 y 5); éste es el caso incluso de algunos estudiantes de 12 años. En los estudios de Gibbs, Majluf, Valdez y Basinger la madurez del juicio moral es alcanzada por adultos con edades bastante mayores a las de los alumnos/as evaluados en 1a presente investigación. Pese a que la mayoría de los sujetos tienen sólo entre 12 y 14 años, los resultados son equiparables a los hallados en un estudio realizado en Lima en un grupo de maestras de educación inicial (Montenegro, 1987).

Con respecto al nivel de pensamiento, se observa que en el área de las operaciones de la logica de las proposiciones un $85 \%$ de los alumnos se ubican en un nivel maduro (niveles IIA F y IIIB F). Este desempeño es mucho mejor al hallado por Majluf (1974) con la misma prueba de Longeot en estudiantes de quinto de secundaria de ambos sexos (con una edad media de 18 años) de colegios particulares de Lima. En el estudio de Majluf sólo el $72 \%$ de los evaluados se ubicaron en un nivel de pensamiento lógico-formal. La diferencia es aún mayor si se consideran sólo los datos de los alumnos de cuarto y quinto de secundaria del colegio "Encinas" (cuyas edades son más cercanas a las del estudio de Majluf), ya que de estos el $92 \%$ se ubicaron en el nivel de pensamiento lógico-formal en la prueba de proposiciones.

De otro lado, los resultados obtenidos confirman los estudios y la teoría que indican que existe una alta correlación positiva entre el nivel intelectual y el nivel moral (hipótesis 3), reforzando las tesis del isomorfismo evolutivo. Sin embargo, ciertos hallazgos parecen contradictorios: en el trabajo de Montenegro (1987), por ejemplo, ninguno de los sujetos que alcanzan el nivel de pensamiento formal se sitúan por debajo del estadío 4 de juicio moral; esto sí ocurre con algunos de los sujetos del presente estudio.

Este estudio también confirma la tesis de la validez universal de las secuencias de etapas y estadíos propuestas por Piaget y por Kohlberg, respectivamente, en sus modelos de desarrollo del pensamiento y del juicio moral (hipótesis 4 
y 5). Esta secuencialidad queda demostrada por la correlación de la edad con el nivel de pensamiento y el desarrollo del juicio moral.

La diferencia no significativa entre varones y mujeres hallada en este estudio puede ser atribuida a que el promedio de edad de las mujeres de la muestra es menor. En muy pocas investigaciones anteriores se han hallado diferencias significativas de género, las que casi siempre han estado asociadas a factores educativos y socio-económicos (Walker, 1984; Snarey, 1985; Valdez, 1985). A esto se puede agregar que, según Gilligan, Kohlberg habría omitido la perspectiva femenina en la formulación de sus dilemas morales y habría basado su descripción de las características del desarrollo moral en resultados obtenidos de una muestra sesgada (Valdez, 1985).

¿Es posible atribuir la superioridad de los puntajes obtenidos en el presente estudio a las caracteristicas del modelo educativo experimental/alternativo aplicado en el colegio estudiado? La contundencia de los resultados, obtenidos en un contexto nacional marcado por el deterioro de la calidad y la eficiencia de la educación, inducen a responder positivamente. Pero la metodología empleada en la investigación no permite formular una conclusión definitiva al respecto.

Cabe señalar, sin embargo, que en estudios previos los alumnos de primaria de este mismo colegio experimental/alternativo han tenido un desempeño significativamente superior al de grupos control en cuanto al dominio de habilidades, conocimientos y actitudes previstos en los programas educativos oficiales (Arellano, 1985) y también respecto al aprendizaje de habilidades para la investigación experimental (Laporta, 1988).

En el estudio de Arellano, realizado a sólo dos años de iniciada la experiencia del "Encinas", de un total de 42 mediciones ( 7 pruebas aplicadas en 6 grados de primaria) 30 promedios fueron más altos en el "Encinas" que en el colegio tomado como control, siendo las diferencias estadísticamente significativas en 11 de las mediciones. Se encontró una notable superioridad de los alumnos del "Encinas" en las pruebas de habilidades de investigación, siendo su ventaja significativa estadísticamente en cinco de los seis grados de primaria (Arellano, 1985).

En el otro estudio mencionado, realizado para validar la propuesta de enseñanza de habilidades de investigación experimental en cuarto y quinto de primaria, se encontraron ganancias mucho mayores de los alumnos del "Encinas" en lo que respecta a estas habilidades en comparación con lo que lograron alumnos de un colegio control sometidos a enseñanza tradicional. Las habilidades incluidas en el programa experimental y evaluadas fueron: medir, describir, identificar variables, formular preguntas, formular hipótesis y diseñar experimentos (Laporta, 1988). 
Por otro lado, diversas investigaciones y experiencias educativas han mostrado las ventajas de una pedagogia activa, personalizada y democrática como la que se aplica en el colegio "José Antonio Encinas". Es significativa la coincidencia de los hallazgos de este estudio con los datos y opiniones de destacados psicólogos y educadores que enfatizan las ventajas de este tipo de diseño pedagógico (Bloom, 1971; Cardemil et al., 1991; Chadwick, 1991; De Prado Díez, 1987; Filp et al., 1986; Piaget, 1974, entre otros).

En la experiencia del colegio "Encinas" sin duda se hallan elementos de diseño pedagógico para una educación mejor que la actual, que pueden ser útiles para quienes quieren convertir las escuelas en lugares que promuevan el desarrollo cognitivo y moral de los alumnos.

\section{Referencias}

Aliaga, J. (1978). Efectos psicológicos de la marginalidad social. Lima: INIDE Arellano, M. 1985. Programa experimental de educación activa: presentación y evaluación con respecto al logro de los objetivos generales de la educación primaria. Informe presentado al Seminario sobre Educación y Sociedad, Lima: FOMCIENCIAS.

Basinger, K.; Gibbs, J. and Fuller, D. (1992). The meaning and measurement of moral development revisited. Ohio State University. Inédito.

Bello, M. (1990). Educar construyendo habilidades. REDES, 1, pp. 20-26.

Bello, M. (1992). Las habilidades como alternativa. Aprender a aprender y a compartir. Ponencia presentada a las III Jomadas Psicopedagogicas del CEDAPP, Lima.

Bloom, B.S. 1971. Taxonomia de los objetivos educacionales. Buenos Aires: Editorial El Ateneo.

Cardemil, C.; Filp, J.; Latorre, M.; Gálvez, G. (1991). Factores que inciden en el mejoramiento de los aprendizajes en la educación básica. Cuadernos de Educación, 208, Santiago: CIDE.

Comisión de Reforma de la Educación. (1970). Reforma de la educación peruana: Informe General. Lima: Ministerio de Educación.

Chadwick, C.B. (1991). Una revolución verde en la educación: las estrategias de aprendizaje. Revista de psicología PUCP, 1, pp. 3-14.

Dammert, J.L. (1985). Renovando la educación primaria. Autoeducación, 12, pp. 25-27.

Delval, J. (1979). Lecturas de psicología del niño (2). Madrid: Alianza Editorial.

De Prado Diez, D. (1987). La ensefianza como desarrollo de aptitudes clave. Escuela para el cambio, 1, pp. 20-27.

Encinas, J. (1932). Un ensayo de escuela nueva en el Perú. Lima: Editorial Minerva. 
Ferreiro, E. (1990). Alternativas a la comprensión del analfabetismo en la región. Revista Tarea, 24.

Filp, J.; Cardemil, C.; Espinola, V. (1986). Disciplina, control social y cambio: estudio de las prácticas pedagógicas en una escuela básica popular. CIDE, Santiago.

Gibbs, J.; Arnold, K.; Morgan, R.; Schawatz, E.; Gavaghan, M.; Tappan, M. (1984). Construction and validation of a multiple choice mesure of moral reasoning. Child Development, 55, pp. 527-536.

Gibbs, J. (1984). Instructions for the use of the Sociomoral Reflection Objective Measure. Ohio: The Ohio State University.

Hersh, R.; Reimer, J.; Paolitto, D. (1984). El crecimiento moral: de Piaget a Kohlberg. Madrid: Narcea S.A.

Holt, J. (1982). El fracaso de la escuela. Madrid: Alianza Editorial.

Laporta, D. (1988). Efectos de un programa de enseñanza de habilidades de investigación experimental en niños de 4to. y 5to. grados de primaria del Programa Educativo "José Antonio Encinas". Tesis de Bachiller en Psicología. Lima: Universidad Peruana Cayetano Heredia.

Majluf, A. (1974). Desarrollo del pensamiento formal proposicional y combinatorio de dos grupos de adolescentes de diferentes estratos socioeconómicos de Lima, Perú. Tesis de Bachiller en Psicología. Lima: UNMSM.

Majluf, A. (1986). Juicio moral de adolescentes de 14 y 16 años, universitarios y adultos de clase socioeconómica media de Lima. Revista de Psicología (PUCP), 4 (1), pp. 73-81.

Ministerio de Educación. (1986). Proyecto educativo nacional. Lima.

Misfud, T. (1985). El pensamiento de Jean Piaget sobre la psicología moral. Presentación crítica. México: Limusa.

Montenegro, E. (1987). Un estudio sobre el nivel del juicio moral y el nivel del pensamiento en un grupo de maestros de educación infantil. Tesis de Maestría. Lima: Universidad Peruana Cayetano Heredia.

Neill, A.S. (1967). Summerhill. México: Fondo de Cultura Económica.

Nisan, M. and Kohlberg, L. (1982). Universality and variation in moral judgment: a longitudinal and cross-sectional study in Turkey. Child Development, 53, pp. 865-876.

Piaget, J. (1973). Los nuevos métodos educativos y sus bases psicológicas. En: Psicología y pedagogía. Barcelona: Editorial Ariel.

Snarey, J. (1985). Cross-cultural universality of social-moral development: a critical review of kohlbergian research. Psychological Bulletin, 97, 2, pp. 202-232.

Skinner, B.F. (1978). El manejo de contingencias en el salon de clases. En Bijou y Rayek (ed.) Análisis conductual aplicado a la instrucción. México: Trillas.

Valdez, M. (1985). Juicio moral en sujetos de 8, 11 y 16 años, de ambos sexos y distinta clase social: un estudio comparativo. Tesis Bachillerato PUCP. Lima.

Walker, L. (1984). Sex differences in the development of moral reasoning: a critical review. Child Development, 55, pp. 677-691. 